\title{
The MAXIMA and MAXIPOL Experiments
}

P.L. Richards, ${ }^{1,3,16}$ M. Abroe, ${ }^{13}$ P. Ade, ${ }^{4}$ A. Balbi, $, 2,3,5$ J. Bock, $, 6,7$ J. Borrill, $, 3,8$ A. Boscaleri, ${ }^{9}$ P. de Bernardis, ${ }^{10}$ J. Collins, ${ }^{1}$ P.G. Ferreira, ${ }^{11,12}$ S. Hanany, ${ }^{3,13}$ V.V. Hristov, ${ }^{7}$ A.H. Jaffe, ${ }^{3}$ B. Johnson, ${ }^{13}$ A.T. Lee, ${ }^{1,2,3}$ T. Matsumu, ${ }^{13}$ P.D. Mauskopf, ${ }^{14}$ C.B. Netterfield, ${ }^{15}$ E. Pascale, ${ }^{9}$ B. Rabii, $, 1,2,3,16$ G.F. Smoot, ${ }^{1,2,3,16}$ R. Stompor, ${ }^{3,16,17}$ C.D. Winant, ${ }^{1,3,16}$ J.H.P. Wu ${ }^{18}$

IDepartment of Physics, University of California, Berkeley, CA 94720-7300, U.S.A.

${ }^{2}$ Division of Physics, Lawrence Berkeley National Laboratory, Berkeley, CA 94720

${ }^{3}$ Center for Particle Astrophysics, University of California, Berkeley, CA 94720-7300, U.S.A.

${ }^{4}$ Queen Mary and Westfield College, London, UK

${ }^{5}$ Dipartimento di Fisica, Università Tor Vergata, Roma, Italy

${ }^{6}$ Jet Propulsion Laboratory, Pasadena, CA, U.S.A.

${ }^{7}$ California Institute of Technology, Pasadena, CA, U.S.A.

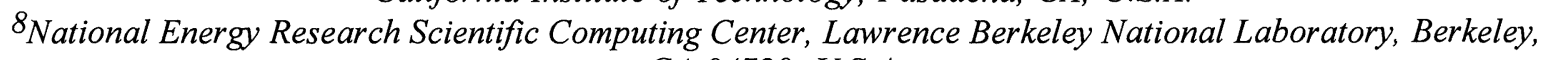
CA 94720, U.S.A.

${ }^{9}$ IROE-CNR, Florence, Italy

10 Dipartimento di Fisica, Università La Sapienza, Rome, Italy

${ }^{11}$ Astrophysics, University of Oxford, Oxford, UK

${ }^{12}$ CENTRA, Instituto Superior Technico, Lisbon, Portugal

${ }^{13}$ School of Physics and Astronomy, University of Minnesota/Twin Cities, Minneapolis, MN, U.S.A.

${ }^{14}$ Department of Physics and Astronomy, University of Wales, Cardiff, UK

${ }^{15}$ Department of Physics and Astronomy, University of Toronto, Canada

${ }^{16}$ Space Sciences Laboratory, University of California, Berkeley, CA 94720-7300, U.S.A.

${ }^{17}$ Copernicus Astronomical Center, Warsaw, Poland

${ }^{18}$ Department of Astronomy, University of California, Berkeley, CA $94720-3411$ U.S.A.

\begin{abstract}
MAXIMA is a balloon-based bolometric experiment to measure the temperature anisotropy of the CMB over spatial frequency range $36 \leq \ell \leq 1235$. The MAXIMA-1 flight produced a 124 square degree temperature anisotropy map with a beam diameter of 10 arcmin. These data have been used to produce a power spectrum which is in excellent agreement with data from BOOMERANG and DASI, but covers a wider range of angular scales. The MAXIMA power spectrum is consistent with the prediction of $\Lambda$ CDM models and has been used to constrain cosmological models. The MAXIMA experiment is described and an outline is given of the contents and significance of papers written by the MAXIMA team. MAXIMA is being modified to measure the polarization anisotropy of the CMB. A brief description of this MAXIPOL experiment is also given.
\end{abstract}

\section{THE MAXIMA-1 EXPERIMENT}

Measurements of the anisotropy of the cosmic microwave background (CMB) can discriminate between cosmological models and determine cosmological parameters with high accuracy (Kamionkowski and Koswoski, 1999) [1]. MAXIMA is a balloon-borne experiment optimized to map the CMB anisotropy over hundreds of square degrees with an angular resolution of $10 \mathrm{arcmin}$ and to produce measurements of the CMB power over a wide range of angular scales corresponding to $36 \leq \ell \leq 1235$. 


\section{Instrumentation}

Lee, et. al. [2] gives a detailed description of the MAXIMA system. It is an off-axis Gregorian telescope mounted on an attitude-controlled balloon platform. The primary mirror rotates so as to scan the telescope beams in azimuth. Re-imaging optics in the receiver cryostat are cooled to $\sim 3 \mathrm{~K}$. The receiver consists of 16 photometers: eight operating at $150 \mathrm{GHz}$, four at $240 \mathrm{GHz}$ and four at $410 \mathrm{GHz}$. All have 10' FWHM beams. The CMB radiation is detected with spider web bolometers (Bock, et. al. [3]) operated at $0.1 \mathrm{~K}$. The bolometers are AC biased to avoid low frequency amplifier noise, and the detector time constants, determined in flight, range from 5 to $13 \mathrm{~ms}$. The combined sensitivity of the eight $150 \mathrm{GHz}$ photometers is $41 \mathrm{microK} \times \mathrm{rt}(\mathrm{sec})$, which is the best of any CMB experiment to date.

The gondola azimuth is driven by a reaction wheel using information from a two-axis magnetometer and three rate gyroscopes. Pointing is reconstructed using a CCD camera bore-sighted to the center of the primary mirror scan and an offset CCD camera that views Polaris. An on-board computer locates the two brightest stars in each CCD field at a $5 \mathrm{~Hz}$ rate.

\section{Observations}

The instrument was launched from the National Scientific Balloon Facility in Palestine, Texas at 1.6 UT 1998 August 2. The CMB anisotropy was measured in two overlapping observations. Each was conducted at a constant elevation angle and consisted of two independent modulations in azimuth: a slow modulation of the entire instrument $+/-5.6$ deg amplitude, $68 \mathrm{sec}$ period and 45 deg elevation for the $1 \mathrm{st}$ scan; $(+/-3.3 \mathrm{deg}, 47$ $\mathrm{sec}, 31 \mathrm{deg}$ elevation for the $2 \mathrm{nd}$ scan) and a fast, saw tooth modulation using the primary mirror $(+/-2$ deg, $0.5 \mathrm{~Hz}$ for both scans). The first observation began at $4.3 \mathrm{UT}$ and lasted 1.7 hours. The second began at 6.0 UT and lasted 1.4 hours. Due to the rotation of the sky, the two scans were cross-linked at an angle of 22 deg. The gondola altitude varied from 38.0 to $38.8 \mathrm{~km}$ during the CMB observations.

\section{Calibration and Beams}

A full beam calibration of the 150 and $240 \mathrm{GHz}$ photometers was obtained from the CMB dipole. The instrument was rotated 100 times with a period of 20 seconds from 3.6 to 4.2 UT. The data from each rotation was fitted to a model including the CMB dipole and Galactic dust emission (Finkbeiner, et. al. [4]). An additional component was found which correlated with the $410 \mathrm{GHz}$ channel signal. This component is consistent with a large angular scale atmospheric contribution and was subtracted. Beam contour maps and were obtained from the Jupiter observation from 7.5 to 8.1 UT. The beam profiles were integrated and used with the angular diameter and brightness temperature of Jupiter (Goldin, et. al. [5]) to calibrate the photometers. A very tight $4 \%$ temperature calibration uncertainty was obtained at 150 and $240 \mathrm{GHz}$. A detailed analysis has been published by Wu et al. [6] of the beam profiles and the procedures required for map making with slightly asymmetric beams.

\section{Data Analysis}

The raw data for each photometer consisted of 1.2 million samples for the first CMB scan and 1 million samples for the second. The data were deglitched, the electronic and bolometric transfer functions deconvolved, and estimates were made of the noise power spectrum of the data stream. It was assumed that the time domain data were dominated by noise and the procedure of Ferreira and Jaffe [6] were used to confirm the validity of this assumption. High and low pass filters were used to eliminate frequencies where there are no appreciable optical signals.

The calibrated time stream data were combined with the attitude pointing solution to produce a maximum likelihood pixelized map of temperature anisotropy and a pixel-pixel noise correlation matrix using the techniques described in Wright [8], Tegmark [9], Bond et. al. [10], and Ferreira and Jaffe [7]. Individual 100 square degree maps were generated from the outputs of four individual photometers. After checking for consistency and verifying that the noise in different maps was uncorrelated, a noise weighted average temperature anisotropy map was produced. The maximum likelihood angular power spectrum $C_{\ell}$ of the map was generated using the MADCAP (Borrill [11]) implementation of the Newton-Raphson iterative 
maximization of the likelihood function following Bond, Jaffe \& Knox [12]. A complete description of the map making procedure has been presented in Stompor et al. [13].

Analysis of data from the MAXIMA-2 flight, launched in June 1999, is in progress. This flight measured over 200 square degrees of the sky. Approximately $25 \%$ of this observing region overlaps with the MAXIMA1 observations.

\section{Foregrounds}

Foreground sources of confusion may include emission from the atmosphere, interstellar dust emission, synchrotron radiation, free-free emission, and point sources. The spectrum of the observed signal is not consistent with that of the atmosphere which has little power at the angular scales measured. The temporal stability of the observed sky structure is inconsistent with an atmospheric or ground-based origin. The ground-based telescope sidelobe measurements (Lee, et. al. [2]) also provides evidence that sidelobe response from the ground or the moon is not significant.

The 100 micron IRAS/DIRBE map (Schlegel et. al. [14]) was extrapolated to the MAXIMA wavebands and cross-correlated with the CMB maps. No correlations large enough to effect CMB power spectrum estimation were found. Using the galactic emission model of Bouchet and Gispert [15] it was found that both Bremstrahlung and Synchrotron emission are expected to contribute fluctuations with a magnitude less than 1 microK at $150 \mathrm{GHz}$. The scan region was chosen to avoid known bright radio point sources and none were detected in our CMB maps. No corrections to the data were required for any source of foreground contamination.

\section{Power Spectrum and Cosmological Parameters}

The MAXIMA map and power spectrum out to $\ell=785$ computed with 5 arcmin pixels and an analysis of the resulting constraints on cosmological parameters were released in Hanany et al [16] and Balbi et al. [17] a few weeks after the first announcement from BOOMERANG [18]. The two data sets are essentially independent, consistent and complementary [16]. BOOMERANG has more statistical weight but MAXIMA extends to higher angular frequencies and has a more accurate calibration. In a joint effort by the two teams, the two data sets were combined by marginalizing over differences in calibration and beam uncertainties and then used to test cosmological models and determine values of cosmological constants in Jaffe et al. [19]. The combined power spectrum clearly defines the first acoustic peak and is consistent with a range of flat $\Lambda \mathrm{CDM}$ models with a relatively large baryon fraction. The data are consistent with the second and third acoustic peaks predicted by the models. The best fit is for a second peak that is somewhat smaller than previously expected [19]. This implies a somewhat larger baryon fraction than that predicted from big bang nucleosynthesis. The data do not demonstrate the existence of a second or third peak because they are also consistent with a horizontal straight line for values of $\ell$ above the first acoustic peak. A seven parameter Bayesian analysis was used to deduce cosmological parameters. The results support the picture of a flat universe, a flat primordial power spectrum, and a baryon fraction of $\sim 5 \%$. When combined with supernova data to break the degeneracy in the $\Omega_{\Lambda} \Omega_{\mathrm{m}}$ plane, the picture emerges of a universe that is $\sim 70 \%$ dark energy, and $\sim 25 \%$ dark matter. If the supernova data are not considered, and data for large scale structure are used to break the degeneracy, the conclusion remains essentially the same. The general agreement obtained for the values of total density and the fractions of dark energy, dark matter and baryons should be considered a triumph for experimental cosmology.

\section{High Resolution Analysis}

The power spectral analysis of the MAXIMA-1 data presented [16,17] out to $\ell=785$ was limited by the 5 arcmin pixels used in the computations. These computations were redone using 23,000 3 arcmin pixels from the fully cross-linked central 60 square degrees of the MAXIMA-1 scans. The resulting power spectrum presented in Lee et al. [20] extends to $\ell=1235$. It is consistent with the previous work and includes the $\ell$ range of the anticipated 3rd acoustic peak. Stompor et al. [21] includes a statistical analysis to show that the point at $\ell=825$ where a third acoustic peak is expected is higher than the surrounding points with $95 \%$ confidence. The existence of multiple acoustic peaks is strong confirmation of inflationary models. Constraints on cosmological parameters based on the high resolution analysis are presented in Stompor et al., [21] where a degeneracy between the primordial slope $n_{S}$ and the optical depth $\tau_{c}$ at the surface of last scattering is discussed. The value of $\Omega_{\mathrm{b}} \mathrm{h}^{2}$ from MAXIMA alone is consistent with the baryon fraction from Big Bang 
nucleosynthesis. The discrepancy noted in Jaffe et al. [19] from the combined MAXIMA BOOMERANG analysis arose because the statistical weight of the BOOMERANG data dominated in the neighborhood of the second acoustic peak.

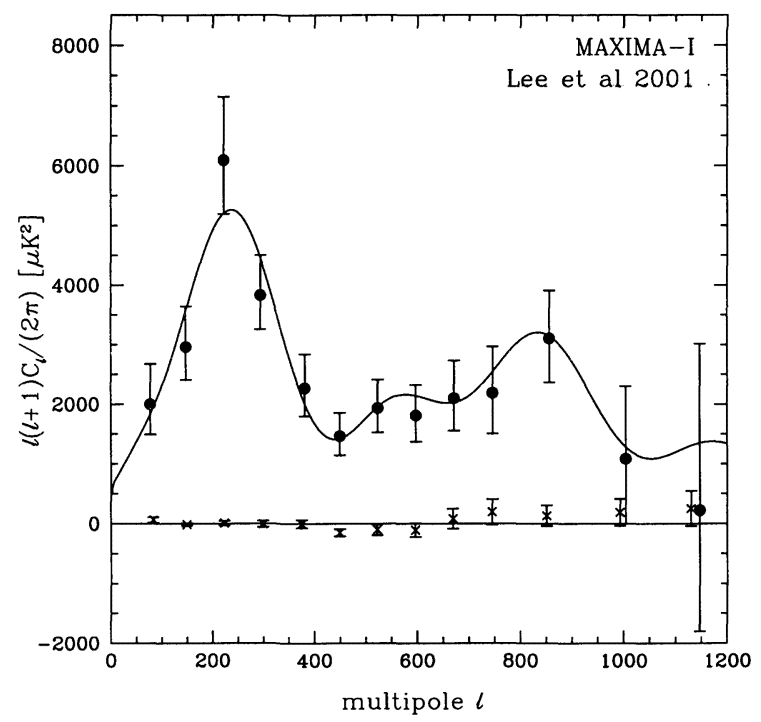

FIGURE 1. Composite angular power spectrum of the CMB anisotropy from the MAXIMA-1 map (filled circles). The points for $l<335$ are from the power spectrum of the full $5^{\prime}$ pixelized map from Hanany et al. [16], and the points for $l>335$ are from the power spectrum of the central region of the $3^{\prime}$ pixelized map. The error bars are $68 \%$ confidence intervals calculated using the offset lognormal likelihood functions of Bond, Jaffe, \& Knox [12]. The solid curve is the best-fit ( $\Lambda \mathrm{CDM})$ inflationary adiabatic cosmology to the Stompor et al. [21] MAXIMA-1 and COBE/DMR power spectrum. The model has $\left.\Omega_{\mathrm{cdm}}, \Omega_{\Lambda}, \tau_{\mathrm{c}}, \mathrm{n}_{\mathrm{S}}, \mathrm{h}\right)=(0.07,0.78,0.0,0.0,1.0,0.53)$ Balbi et al. [17]. The crosses are the estimated power spectrum of the difference between two independent maps, the first given by one of the three photometers and the other from the sum of the other two. The data in this figure are the same as those from Lee et al. [20], except for the expanded scale. The theoretical fit has been changed to that of Stompor et al. [21].

\section{Gaussianity}

Gaussianity of cosmological perturbations in the universe is one of the key predictions of standard inflation, but not of other models of structure formation such as cosmic defects. Tests for Gaussianity have been carried out using many methods for several CMB data sets, especially the COBE DMR map. All tests of the DMR data indicate Gaussianity, except for two that may have non-cosmological origins. The DMR data are not ideal for these tests since they probe scales larger than the causal horizon and thus combine uncorrelated perturbations. The central-limit theorem shows that non-Gaussianity will be erased on such large angular scales.

$\mathrm{Wu}$ et al. [22] have used the methods of moments, the Kolmogorov test, the $\mathrm{X}^{2}$ test and Minkowski functionals in eigen, real, Wiener-filtered and signal-whitened spaces to test the MAXIMA-1 data. The data are consistent with Gaussianity. This result is consistent with standard cosmological inflation and place limits on the existence of cosmic defects. It also justifies the methods of data analysis used to produce maps, power spectra and cosmological parameters, which are valid only for Gaussian fluctuations. In a related work, Santos, et al. [23] investigate the bispectrum of the MAXIMA-1 data and conclude that the results are consistent with a Gaussian sky. 


\section{MAXIPOL}

The MAXIMA photometer is being converted to a polarimeter to measure the polarization anisotropy of the CMB. The conversion is simple to describe, but difficult to carry out in practice. Grid polarizers are being placed in front of the photometer horns in the focal plane. A cold anti-reflection coated half-wave plate is located at the Lyot stop and rotated at a few $\mathrm{Hz}$, which is fast compared with the gondola scan rate. The direction and strength of a polarized signal from the sky will then be obtained by synchronous demodulation of the data. Relatively small regions of the sky with low dust contamination will be mapped deeply so as to detect the E-mode polarization of the CMB. A turnaround flight is planned for Ft. Sumner, N.M. in spring 2002.

\section{ACKNOWLEDGMENTS}

We thank Danny Ball and the other staff at NASA's National Scientific Balloon Facility in Palestine, TX for their outstanding support of the MAXIMA program. MAXIMA is supported by NASA Grants NAG53941, NAG5-6552, NAG5-4454, GSRP-031, and GSRP-032, and by the NSF through the Center for Particle Astrophysics at UC Berkeley, NSF Cooperative Agreement AST-9120005, and KDI Grant 9872979. The data analysis used resources of the National Energy Research Scientific Computing Center which is supported by the Office of Science of the U.S. Department of Energy under Contract No. DE-AC03-76SF00098, and the resources of the Minnesota Supercomputing Institute. PA acknowledges support from PPARC rolling grant, UK.

\section{REFERENCES}

1. Kamionkowski, M., and Kosowsky, A., The Cosmic Microwave Background and Particle Physics, Ann. Rev. Nucl. Part. Sci. 49, 77-123 (1999).

2. Lee, A. T., et. al., MAXIMA: An Experiment to Measure Temperature Anisotropy in the Cosmic Microwave Background, in L. Maiani, F. Melchiorri and N. Vittorio (eds.), AIP Conf. Proc. 476, pp 224-236.Proceedings of 3K Cosmology Conference, Rome, Italy, astro-ph/9903249 (1998).

3. Bock, J. J., et al., A Novel Bolometer for Infrared and Millimeter-Wave Astrophysics, Space Sciences Reviews 74, 229-235 (1995).

4. Finkbeiner, D. P., et. al., Extrapolation of Galactic Dust Emission at 100 Microns to CMBR Frequencies Using FIRAS, $A p$. J. 524, 867 (1999)

5. Goldin, A. B., et. al., Whole Disk Observations of Jupiter, Saturn and Mars in Millimeter-Submillimeter Bands, Ap.J.Lett. 488, L161, (1997).

6. Wu, J.H.P., et al., Asymmetric Beams in Cosmic Microwave Background Anisotropy Experiments, Astroph/0007212, Ap. J. Suppl. 132, 1 (2001).

7. Ferreira, P. G., and Jaffe, A. H., Simultaneous Estimation of Noise and Signal in Cosmic Microwave Background Experiments, Monthly Notices of the Royal Astron. Soc. 312, 89 (2000).

8. Wright, E., Scanning and Mapping Strategies for CMB Experiments, Report UCLA-ASTRO-ELW-96-03, (1996) presented at the IAS CMB Data Analysis Workshop in Princeton, astro-ph/9612006.

9. Tegmark M., How to Make Maps from CMB Data Without Losing Information, astro-ph/9611130, Ap. J. 480, 87 (1997)

10. Bond, J. R., et. al., Computing Challenges of the Cosmic Microwave Background, Computing in Science and Engineering 1, 21 (1999).

11. Borrill, J, MADCAP-The Microwave Anisotropy Dataset Computational Package, Proceedings of the Fifth European SGI/Cray MPP Workshop, astro-ph/9911389 (1999).

12. Bond, J. R., Jaffe, A. H., and Knox, L., Estimating the Power Spectrum of the Cosmic Microwave Background, Phys. Rev. D57, 2117 (1998).

13. Stompor, R., et al., Making Maps of the Cosmic Microwave Background: The MAXIMA Example, astroph/0106451, Phys. Rev. D (to be published).

14. Schlegel D. J., et. al., Maps of Dust Infrared Emission for Use in Estimation of Reddening and Cosmic Microwave Background Radiation Foregrounds, Ap. J. 500, 525 (1998), and Application of SFD Dust Maps to Galaxy Counts and CMB Experiments, in S. Colombi and Y. Mellier (eds.) Wide Field Surveys in Cosmology, (in press) astro-ph/9809230.

15. Bouchet, R. B., and Gispert, R., Foregrounds and CMB Experiments: I. Semi-Analytical Estimates of Contamination, astro-ph/9903176, New Astron. 4, 443-479 (1999).

16. Hanany, S., et al., MAXIMA-1: A Measurement of the Cosmic Microwave Background Anisotropy on Angular Scales of 10 Arcminutes to 5 Degrees, Ap. J. Lett. 545, L5 (2000). 
17. Balbi, A. et al., Constraints on Cosmological Parameters from MAXIMA-1, astro-ph/0005124, Ap. J. 545, L1 (2000).

18. de Bernardis, P., et al., A Flat Universe from High-Resolution Maps of the Cosmic Microwave Background Radiation, Nature 404, 955-959 (2000).

19. Jaffe, A.H., et al. Cosmology from MAXIMA-1, BOOMERANG, and COBE/DMR Cosmic Microwave Background Observations, Phys. Rev. Lett. 86, 3475 (2001).

20. Lee, A.T., et al., A High Spatial Resolution Analysis of the MAXIMA-1 Cosmic Microwave Background Anisotropy Data, astro-ph/0104459, Ap. J. Letters (to be published) .

21. Stompor, R., et al., Cosmological Implications of the MAXIMA-1 High Resolution Cosmic Microwave Background Anisotropy Measurement, astro-ph/0105062, Ap. J. Lett. (to be published)

22. Wu, J.H.P., et al., Tests for Gaussianity of the MAXIMA-1 CMB Map, astro-ph/0104248, Phys. Rev. Lett. (submitted).

23. Santos, M.G., et al., An Estimate of the Cosmological Bispectrum from the MAXIMA-1 CMB Map, astroph/0107588, Phys. Rev. Lett. (submitted). 